J. Korean Math. Soc. 52 (2015), No. 4, pp. 853-868

http://dx.doi.org/10.4134/JKMS.2015.52.4.853

\title{
GROSSBERG-KARSHON TWISTED CUBES AND BASEPOINT-FREE DIVISORS
}

\author{
Megumi Harada and Jihyeon Jessie Yang
}

\begin{abstract}
Let $G$ be a complex semisimple simply connected linear algebraic group. The main result of this note is to give several equivalent criteria for the untwistedness of the twisted cubes introduced by Grossberg and Karshon. In certain cases arising from representation theory, Grossberg and Karshon obtained a Demazure-type character formula for irreducible $G$-representations as a sum over lattice points (counted with sign according to a density function) of these twisted cubes. A twisted cube is untwisted when it is a "true" (i.e., closed, convex) polytope; in this case, Grossberg and Karshon's character formula becomes a purely positive formula with no multiplicities, i.e., each lattice point appears precisely once in the formula, with coefficient +1 . One of our equivalent conditions for untwistedness is that a certain divisor on the special fiber of a toric degeneration of a Bott-Samelson variety, as constructed by Pasquier, is basepoint-free. We also show that the strict positivity of some of the defining constants for the twisted cube, together with convexity (of its support), is enough to guarantee untwistedness. Finally, in the special case when the twisted cube arises from the representation-theoretic data of $\lambda$ an integral weight and $\underline{w}$ a choice of word decomposition of a Weyl group element, we give two simple necessary conditions for untwistedness which is stated in terms of $\lambda$ and $\underline{w}$.
\end{abstract}

\section{Introduction}

Constructing a combinatorial model for a basis of a representation is a fruitful technique in modern representation theory, as exhibited by the famous theory of crystal bases and string polytopes. Another well-known example from toric geometry is the bijective correspondence between the lattice points in the moment polytope of a nonsingular projective toric variety $X$ with a basis consisting of $T$-weight vectors of the space $H^{0}(X, L)$ of holomorphic sections of the prequantum line bundle $L$ over $X$. These two examples are linked via toric degenerations: Kaveh [6] recently showed that string polytopes can be obtained as Okounkov bodies of flag varieties $G / B$, and using results of Anderson [2]

Received October 7, 2014.

2010 Mathematics Subject Classification. Primary 14M25, 20G05; Secondary 14C20.

Key words and phrases. twisted cubes, Demazure character formula, Bott-Samelson variety, toric variety, toric divisor. 
one can show that in this case there is a toric degeneration of $G / B$ to a toric variety $X$ whose corresponding polytope is the string polytope.

In much earlier work (from the 1990s) Grossberg and Karshon [4] also constructed degenerations of complex structures from Bott-Samelson varieties to toric varieties (specifically, Bott towers) and consequently obtained a Demazure-type character formula for irreducible representations of complex semisimple (simply-connected) linear algebraic groups $G$. Their character formula can be combinatorially interpreted in terms of twisted cubes (cf. Definition 1.1). These twisted cubes are combinatorially simpler than general string polytopes but they are not "true" polytopes in the sense that their faces may have various angles and the intersection of faces may not be a face (cf. [4, $\S 2.5$ and Figure 1 therein]), and (the support of the) twisted cube may be neither closed nor convex. An example is shown in Figure 1.1. In particular, the Grossberg-Karshon character formula takes a sum over a set of integer lattice points, where a summand may appear with either a plus or minus sign [4, Theorem 6 and Remark 3.22]. In this sense, the Grossberg-Karshon character formula is not, in general, a purely combinatorial 'positive' formula.

The main result of this note, recorded in Theorem 2.4, gives several equivalent conditions for the Grossberg-Karshon twisted cubes to be untwisted (cf. Definition 2.2), i.e., it is a "true" (closed, convex) polytope. In the representation-theoretic applications of Grossberg and Karshon, this corresponds to the case when the Grossberg-Karshon character formula is a 'positive' formula. One of our equivalent conditions can be stated naturally in terms of the toric variety $X(\mathbf{c})$ obtained as the special fiber of a toric degeneration of Bott-Samelson varieties as constructed by Pasquier [8] (which was in turn motivated from the degeneration of complex structures from a Bott-Samelson variety to a Bott tower given in [4]). More specifically, the condition is that a certain torusinvariant divisor $D(\mathbf{c}, \ell)$ on the toric variety $X(\mathbf{c})$ is basepoint-free. By some standard results in toric geometry, the basepoint-free-ness of a divisor can also be stated in terms of the Cartier data of the divisor, and this in turn provides us with computationally efficient methods for determining the untwistedness of the Grossberg-Karshon twisted cube. We note that the relationship between untwistedness and basepoint-free-ness seems to have already been known by experts (see e.g. the comments in $[1, \S 6]$ ). However, our computationally effective characterization of untwistedness in terms of the Cartier data of $D(\mathbf{c}, \ell)$ depends on an explicit description of the line bundle $\mathcal{L}_{0}$ (over the special fiber of the toric degeneration) as $\mathcal{O}(D(\mathbf{c}, \ell))$, i.e., the line bundle corresponding to the divisor $D(\mathbf{c}, \ell)$, as well as a concrete computation of $D(\mathbf{c}, \ell)$. As far as we are aware, these results do not appear in the previous literature.

In Section 3 we also record the observation that the convexity of the twisted cube, and the strict positivity of a certain subset of the constants used in the definition of the twisted cube, are sufficient to guarantee its untwistedness. Finally, in Section 4 we give two simple necessary conditions for the untwistedness of the Grossberg-Karshon twisted cube $(C, \rho)$ when it arises from the 
representation-theoretic data of a weight $\lambda \in \mathfrak{t}_{\mathbb{Z}}^{*}$ and a choice of reduced word decomposition $\underline{w}$ for an element $w$ in the Weyl group of $G$. The two necessary conditions are not sufficient, as can be seen by a simple example (cf. Example 4.3); a full discussion of necessary and sufficient conditions for untwistedness, stated in terms of the representation theoretic data, is in [5].

Finally, we remark that Kiritchenko recently has defined divided difference operators $D_{i}$ on polytopes and, using these $D_{i}$ inductively and a fixed choice of reduced word decomposition for the longest element in the Weyl group of $G$, she constructs (possibly virtual) polytopes whose lattice points encode the character of irreducible $G$-representations [7, Theorem 3.6]. Kiritchenko's virtual polytopes simultaneously generalize many (virtual) polytopes already known in representation theory, including the Grossberg-Karshon twisted polytopes and the well-known Gel'fand-Cetlin polytopes (which are special cases of string polytopes). Kiritchenko additionally shows that Gel'fand-Cetlin polytopes degenerate to certain Grossberg-Karshon twisted polytopes via a sequence of 'string spaces' [7, §4]. As in the case of Grossberg and Karshon, since Kiritchenko's polytopes may be virtual, her character formula is also not necessarily 'purely positive' in the sense that some coefficients may appear with a minus sign. Therefore, it is of interest (see also her discussion in $[7, \S 3.3]$ ) to determine when Kiritchenko's virtual polytopes are in fact 'true' polytopes. The results of this note may be viewed as a partial answer to this more general question.

Acknowledgements. The first author is partially supported by an NSERC Discovery Grant (Individual), an Ontario Ministry of Research and Innovation Early Researcher Award, a Canada Research Chair (Tier 2) award, and a Japan Society for the Promotion of Science Invitation Fellowship for Research in Japan (Fellowship ID L-13517). Both authors thank the Osaka City University Advanced Mathematics Institute for its hospitality while part of this research was conducted.

\section{Background}

\subsection{Twisted cubes}

We begin by recalling the definition of twisted cubes given by Grossberg and Karshon $[4, \S 2.5]$. Fix a positive integer $n$. A twisted cube is a pair $(C(\mathbf{c}, \ell), \rho)$ where $C(\mathbf{c}, \ell)$ is a subset of $\mathbb{R}^{n}$ and $\rho: \mathbb{R}^{n} \rightarrow \mathbb{R}$ is a density function with support precisely $C(\mathbf{c}, \ell)$. Here $\mathbf{c}=\left\{c_{i j}\right\}_{1 \leq i<j \leq n}$ is a collection of integers and $\ell=\left\{\ell_{1}, \ell_{2}, \ldots, \ell_{n}\right\}$ is a collection of real numbers. In order to simplify the notation in what follows, we define the following functions on $\mathbb{R}^{n}$ using the usual coordinates $x=\left(x_{1}, \ldots, x_{n}\right)$ :

$$
A_{n}(x)=A_{n}\left(x_{1}, \ldots, x_{n}\right)=\ell_{n},
$$




$$
A_{j}(x)=A_{j}\left(x_{1}, \ldots, x_{n}\right)=\ell_{j}-\sum_{k=j+1}^{n} c_{j k} x_{k} \text { for all } 1 \leq j \leq n-1 .
$$

Notice that $A_{n}(x)$ is in fact a constant function and that $A_{j}(x)$ for $1 \leq j \leq n-1$ is a linear function depending only of the variables $x_{j+1}, \ldots, x_{n}$. In order to emphasize this, we will sometimes write $A_{j}(x)=A_{j}\left(x_{j+1}, \ldots, x_{n}\right)$. We also define a function $\operatorname{sgn}: \mathbb{R} \rightarrow\{ \pm 1\}$ by $\operatorname{sgn}(x)=1$ for $x<0$ and $\operatorname{sgn}(x)=-1$ for $x \geq 0$. We now give a sequence of $n$ logical statements, each of which involves a logical "or". Specifically, for $k$ with $1 \leq k \leq n$ we say (S-k) is the statement

$$
\begin{aligned}
A_{k}(x) & =A_{k}\left(x_{k+1}, \ldots, x_{n}\right)<x_{k}<0 \text { or } \\
0 \leq x_{k} \leq A_{k}(x) & =A_{k}\left(x_{k+1}, \ldots, x_{n}\right) .
\end{aligned}
$$

Note that the condition (S-k) depends only on the last $n-k+1$ variables $\left(x_{k}, \ldots, x_{n}\right)$.

With the above in place we can give the definition.

Definition 1.1. With notation as above, let $C(\mathbf{c}, \ell)$ denote the following subset of $\mathbb{R}^{n}$ :

$(1.2)$

$$
\begin{aligned}
C(\mathbf{c}, \ell) & :=\left\{x=\left(x_{1}, \ldots, x_{n}\right) \in \mathbb{R}^{n} \mid x \text { satisfies condition }(\mathrm{S}-\mathrm{k}) \text { for all } 1 \leq k \leq n\right\} \\
& \subseteq \mathbb{R}^{n} .
\end{aligned}
$$

Moreover we define a density function $\rho: \mathbb{R}^{n} \rightarrow \mathbb{R}$ by

$$
\rho(x)= \begin{cases}(-1)^{n} \prod_{k=1}^{n} \operatorname{sgn}\left(x_{k}\right) & \text { if } x \in C(\mathbf{c}, \ell) \\ 0 & \text { else. }\end{cases}
$$

We call the pair $(C(\mathbf{c}, \ell), \rho)$ the twisted cube associated to $\mathbf{c}$ and $\ell$. To simplify notation we sometimes write $C$ instead of $C(\mathbf{c}, \ell)$.

Remark 1.2 .

- Our definition is slightly different from the one given in $[4, \S 2.5]$ but the two definitions may be identified via the coordinate change $\left(x_{1}, \ldots, x_{n}\right)$ $\mapsto\left(-x_{1}, \ldots,-x_{n}\right)$.

- Grossberg and Karshon refer to the "standard" twisted cube, but we will omit the word "standard" from our terminology.

- In the representation-theoretic applications we have in mind (see Section 4), the constants $\ell_{j}$ are integers.

As the following examples show, a twisted cube may not be a cube in the standard sense. In particular, the set $C=C(\mathbf{c}, \ell)$ may be neither convex nor closed.

Example 1.3. Let $n=2$ and let $\ell=\left(\ell_{1}=3, \ell_{2}=5\right)$ and $\mathbf{c}=\left\{c_{12}=1\right\}$. Then $C=\left\{\left(x_{1}, x_{2}\right) \in \mathbb{R}^{2} \mid 0 \leq x_{2} \leq 5\right.$ and $\left(3-x_{2}<x_{1}<0\right.$ or $\left.\left.0 \leq x_{1} \leq 3-x_{2}\right)\right\}$.

See Figure 1.1. The value of the density function $\rho$ is recorded within each region. 


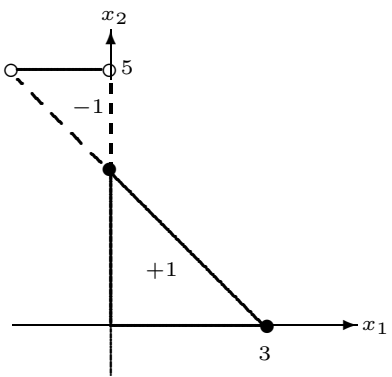

FiguRE 1.1

Note in particular that $C$ does not contain the points $\left\{\left(0, x_{2}\right) \mid 3<x_{2}<5\right\}$ and the points $\left\{\left(x_{1}, x_{2}\right) \mid 3<x_{2}<5\right.$ and $\left.x_{1}=3-x_{2}\right\}$, so $C$ is not closed, and it is also not convex.

Example 1.4. Let $n=2$ and let $\ell=\left(\ell_{1}=-7, \ell_{2}=5\right)$ and $\mathbf{c}=\left\{c_{12}=-1\right\}$. Then

$C=\left\{\left(x_{1}, x_{2}\right) \in \mathbb{R}^{2} \mid 0 \leq x_{2} \leq 5\right.$ and $\left(-7+x_{2}<x_{1}<0\right.$ or $\left.\left.0 \leq x_{1} \leq-7+x_{2}\right)\right\}$.

See Figure 1.2. Here $C$ is convex but not closed.

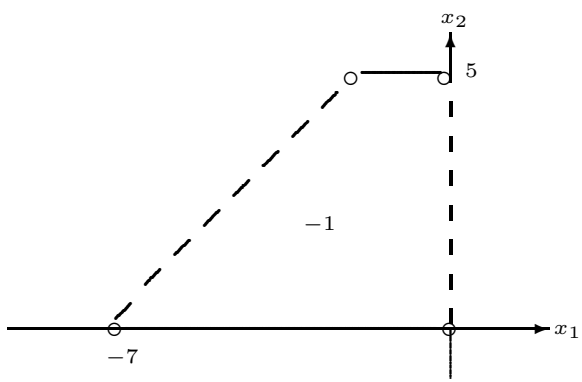

FIGURE 1.2

The goal of the present manuscript is to give conditions under which the Grossberg-Karshon twisted cube associated to $\ell$ and $\mathbf{c}$ is "untwisted", i.e., the support $C=C(\mathbf{c}, \ell)$ is a convex polytope (in particular $C$ is closed), and the density function $\rho$ is constant and equal to 1 on $C$ and 0 elsewhere. In this direction it is useful to note that, roughly speaking, the "twistedness" of $(C, \rho)$ is a consequence of the logical "or" present in the statements (S-k). If it happens that the constants $\mathbf{c}=\left\{c_{i j}\right\}$ and $\ell=\left\{\ell_{1}, \ldots, \ell_{n}\right\}$ are such that the only way to satisfy (1.2) is to always satisfy the inequality in the second statement of (S-k) (i.e., $0 \leq x_{k} \leq A_{k}(x)$ ), then $C=C(\mathbf{c}, \ell)$ is an intersection of closed half-spaces and is a closed convex polytope. The definition below formalizes this simple idea.

Definition 1.5. Let $n, \mathbf{c}, \ell$ and $A_{j}$ be as above. We say that $\mathbf{c}, \ell$ satisfy condition $(\mathrm{P})$ if 
(P-n) $\ell_{n} \geq 0$

and for every integer $k$ with $1 \leq k \leq n-1$, the following statement, which we refer to as condition (P-k), holds:

$(\mathrm{P}-\mathrm{k})$ if $\left(x_{k+1}, \ldots, x_{n}\right)$ satisfies

$$
\begin{aligned}
& 0 \leq x_{n} \leq A_{n}=\ell_{n} \\
& 0 \leq x_{n-1} \leq A_{n-1}\left(x_{n}\right) \\
& \quad \quad \quad \\
& 0 \leq x_{k+1} \leq A_{k+1}\left(x_{k+2}, \ldots, x_{n}\right)
\end{aligned}
$$

then $A_{k}\left(x_{k+1}, \ldots, x_{n}\right) \geq 0$.

In particular, condition $(\mathrm{P})$ holds if and only if the conditions $(\mathrm{P}-1)$ through (P-n) all hold.

It is not difficult to see that if $\mathbf{c}, \ell$ satisfy condition $(\mathrm{P})$, then $C=C(\mathbf{c}, \ell)$ is the closed convex polytope defined by

$$
\begin{aligned}
\left\{x \in \mathbb{R}^{n} \mid 0\right. & \leq x_{n} \leq A_{n}=\ell_{n}, 0 \leq x_{n-1} \leq A_{n-1}\left(x_{n}\right), \ldots, \\
0 & \left.\leq x_{1} \leq A_{1}\left(x_{2}, \ldots, x_{n}\right)\right\}
\end{aligned}
$$

In what follows, we frequently find it useful to argue inductively on the size of $n$. For this purpose it is convenient to define the following.

Definition 1.6. For a fixed $n, \mathbf{c}, \ell$ and fixed $1 \leq k \leq n$, we define a subset $C(k)$ of $\mathbb{R}^{k}$ defined to be the set of points satisfying conditions (S-n), (S- $\left.(\mathrm{n}-1)\right)$, $\ldots, \mathrm{S}(\mathrm{n}-\mathrm{k}+1)$. Here we identify $\mathbb{R}^{k}$ with the subspace of $\mathbb{R}^{n}$ corresponding to the variables $\left(x_{n-k+1}, \ldots, x_{n}\right)$.

The following is immediate.

Lemma 1.7. Let $n$ be a positive integer and $\mathbf{c}, \ell$ fixed constants. For any $k$ with $1 \leq k \leq n$, the projection $\pi_{k}: \mathbb{R}^{n} \rightarrow \mathbb{R}^{k}$ given by $\left(x_{1}, \ldots, x_{n}\right) \mapsto$ $\left(x_{n-k+1}, \ldots, x_{n}\right)$ induces a surjective map $C=C(\mathbf{c}, \ell) \rightarrow C(k)$. In particular, the image $\pi_{k}(C)$ is precisely equal to $C(k)$.

\subsection{Divisors, Cartier data, and polytopes}

In this section we briefly recall a construction given by Pasquier [8] of a non-singular toric variety $X(\mathbf{c})$ and a torus-invariant divisor $D(\mathbf{c}, \ell)$ on $X(\mathbf{c})$, associated to the data $\mathbf{c}=\left\{c_{i j}\right\}$ and $\ell=\left\{\ell_{j}\right\}$. As mentioned in the introduction, the variety $X(\mathbf{c})$ arises as the special fiber of a toric degeneration of a Bott-Samelson variety. Indeed, Pasquier's construction of this toric degeneration is the algebro-geometric version of the degeneration of complex structures given by Grossberg and Karshon in [4]. However, this perspective is not necessary in this paper; here we only need the explicit formula for the relevant toric-invariant divisor $D(\mathbf{c}, \ell)$. 
Let $n$ be a fixed positive integer and $\mathbf{c}=\left\{c_{i j}\right\}$ and $\ell=\left\{\ell_{k}\right\}$ be collections of constants as in Section 1. In this section we assume the $\ell_{k}$ are integers for all $k$. Let $\left\{e_{1}^{+}, \ldots, e_{n}^{+}\right\}$denote the standard basis of $\mathbb{R}^{n}$ and let $\mathbb{Z}^{n}$ be the standard lattice in $\mathbb{R}^{n}$ generated by the $e_{j}^{+}$. Define vectors $e_{j}^{-}$for $j=1, \ldots, n$ by the formula

$$
e_{j}^{-}:=-e_{j}^{+}-\sum_{k>j} c_{j k} e_{k}^{+} .
$$

Note that the $e_{j}^{-}$depend only on $\mathbf{c}=\left\{c_{i j}\right\}$ and not on $\ell$.

For basic background on toric varieties we refer the reader to [3]. Let $\Sigma_{\mathbf{c}}$ denote the fan consisting of cones generated by subsets of $\left\{e_{1}^{+}, \ldots, e_{n}^{+}, e_{1}^{-}, \ldots, e_{n}^{-}\right\}$ which do not contain any subsets of the form $\left\{e_{j}^{+}, e_{j}^{-}\right\}$. From this it easily follows that the set of maximal ( $n$-dimensional) cones $\Sigma_{\mathbf{c}}(n)$ of $\Sigma_{\mathbf{c}}$ is in 1-1 correspondence with the vectors $\left\{\sigma=\left(\sigma_{1}, \ldots, \sigma_{n}\right) \mid \sigma_{i} \in\{+,-\}\right\}$ via the map $\sigma \mapsto$ Cone $\left\{e_{1}^{\sigma_{1}}, \ldots, e_{n}^{\sigma_{n}}\right\}$. In particular, $\left|\Sigma_{\mathbf{c}}(n)\right|=2^{n}$. Let $X(\mathbf{c})=X\left(\Sigma_{\mathbf{c}}\right)$ denote the toric variety associated to the fan $\Sigma_{\mathbf{c}}$. Since each cone is generated by a $\mathbb{Z}$-basis, the fan $\Sigma_{\mathbf{c}}$ is smooth and thus $X(\mathbf{c})$ is smooth $[3$, Theorem 3.1.19]. Next, for each $j, 1 \leq j \leq n$, let $D_{e_{j}^{-}}$be the torus-invariant divisor on $X(\mathbf{c})$ corresponding to the ray spanned by $e_{j}^{-}[3, \S 4.1]$. We now define the torus-invariant divisor $D(\mathbf{c}, \ell)$ on $X(\mathbf{c})$ by

$$
D(\mathbf{c}, \ell):=\sum_{j=1}^{n} \ell_{j} D_{e_{j}^{-}} .
$$

Since $X(\mathbf{c})$ is non-singular, the divisor $D(\mathbf{c}, \ell)$ is Cartier [3, Theorem 4.0.22]. In what follows we give an explicit computation of the so-called Cartier data $\left\{m_{\sigma}\right\}_{\sigma \in \Sigma_{\mathbf{c}}(n)}$ of $D(\mathbf{c}, \ell)$ [3, Theorem 4.2.8]. We first set some notation. Here we view $\mathbb{R}^{n}$ as $N \otimes \mathbb{R}$ where $N \cong \mathbb{Z}^{n}$ is the free abelian group of one-parameter subgroups of a torus $\left(\mathbb{C}^{*}\right)^{n}$. Let $M \cong \mathbb{Z}^{n}$ denote the free abelian group of characters of the same torus and let $\langle\cdot, \cdot\rangle: M \times N \rightarrow \mathbb{Z}$ denote the usual bilinear pairing between characters and one-parameter subgroups [3, §1.1]. (With the standard identifications of $M$ and $N$ with $\mathbb{Z}^{n}$, the bilinear pairing above is just the usual inner product.) For any fan $\Sigma$, let $\Sigma(1)$ denote the set of 1dimensional cones in $\Sigma$, and for $\rho \in \Sigma(1)$ let $D_{\rho}$ denote the torus-invariant divisor corresponding to $\rho[3, \S 4.1]$. Further, we let $u_{\rho}$ be the minimal generator of $N \cap \rho$. Let $\Sigma_{\max }$ denote the set of maximal cones, and for $\sigma \in \Sigma_{\max }$ let $\sigma(1)$ denote the set of rays in $\sigma$.

We quote the following from [3].

Theorem 1.8 (cf. [3, Theorem 4.2.8]). Let $\Sigma$ be a fan, $X(\Sigma)$ the toric variety associated to $\Sigma$, and $D=\sum_{\rho} a_{\rho} D_{\rho}$ a divisor. Then the following are equivalent:

- $D$ is Cartier.

- For each $\sigma \in \Sigma_{\max }$, there exists an element $m_{\sigma} \in M$ with $\left\langle m_{\sigma}, u_{\rho}\right\rangle=$ $-a_{\rho}$ for all $\rho \in \sigma(1)$. 
A collection $\left\{m_{\sigma}\right\}$ satisfying the above conditions is called the Cartier data of $D(\mathbf{c}, \ell)$.

In our case, the maximal cones are precisely the $n$-dimensional cones $\Sigma_{\mathbf{c}}(n)$, which in turn are in 1-1 correspondence with the set of $\sigma$ in $\{+,-\}^{n}$. Using this identification we may refer to $\sigma=\left(\sigma_{1}, \ldots, \sigma_{n}\right) \in\{+,-\}^{n}$ as a maximal cone. The following lemma gives an explicit computation of the Cartier data $\left\{m_{\sigma}\right\}$ of $D(\mathbf{c}, \ell)$.

Lemma 1.9. Let $\sigma=\left(\sigma_{1}, \ldots, \sigma_{n}\right) \in\{+,-\}^{n}$ be a maximal cone in $\Sigma_{\mathbf{c}}(n)$. Then the associated Cartier data $m_{\sigma}=\left(m_{\sigma, 1}, \ldots, m_{\sigma, n}\right) \in \mathbb{Z}^{n}$ of $D(\mathbf{c}, \ell)$ is given by the formula

$$
m_{\sigma, j}= \begin{cases}0 & \text { if } \sigma_{j}=+ \\ A_{j}\left(m_{\sigma, j+1}, \ldots, m_{\sigma, n}\right) & \text { if } \sigma_{j}=-\end{cases}
$$

for all $1 \leq j \leq n$.

Proof. By Theorem 1.8, the vector $m_{\sigma} \in \mathbb{Z}^{n}$ must satisfy the condition

$$
\left\langle m_{\sigma}, u_{\rho}\right\rangle=-a_{\rho}
$$

for all 1-dimensional rays $\sigma(1)$ in $\sigma$. Recall that $\sigma=\left(\sigma_{1}, \ldots, \sigma_{n}\right) \in\{+,-\}^{n}$ is identified with Cone $\left\{e_{1}^{\sigma_{1}}, \ldots, e_{n}^{\sigma_{n}}\right\}$ and that $D(\mathbf{c}, \ell)=\sum_{j=1}^{n} \ell_{j} D_{e_{j}^{-}}$. Thus $m_{\sigma}$ is required to satisfy the $n$ equations

$$
\left\langle m_{\sigma}, e_{j}^{\sigma_{j}}\right\rangle= \begin{cases}0 & \text { if } \sigma_{j}=+ \\ -\ell_{j} & \text { if } \sigma_{j}=-\end{cases}
$$

In particular, since $e_{j}^{+}$is a standard basis vector, we see that if $\sigma_{j}=+$, then $m_{\sigma, j}=0$. If $\sigma_{j}=-$, then from the definition of the $e_{j}^{-}$in (1.5) it follows that $m_{\sigma, j}=A_{j}\left(m_{\sigma, j+1}, \ldots, m_{\sigma, n}\right)$.

Next recall from $[3, \S 6.1]$ that there exists a polytope $P_{D}$ associated to any torus-invariant Cartier divisor $D=\sum_{\rho} a_{\rho} D_{\rho}$ on a toric variety. Specifically, we define (cf. [3, Equation (6.1.1)]):

$$
P_{D}:=\left\{m \in M \otimes \mathbb{R} \mid\left\langle m, u_{\rho}\right\rangle \geq-a_{\rho} \text { for all } \rho \in \Sigma(1)\right\} .
$$

In our setting, the 1-dimensional cones are those spanned by $n$ primitive vectors $\left\{e_{1}^{\sigma_{1}}, \ldots, e_{n}^{\sigma_{n}}\right\}$, so we obtain

$$
P_{D(\mathbf{c}, \ell)}=\left\{x \in M \otimes \mathbb{R} \cong \mathbb{R}^{n} \mid 0 \leq x_{j} \leq A_{j}(x) \text { for all } 1 \leq j \leq n\right\}
$$

by using the definition of the $e_{j}^{-}(j=1, \ldots, n)$. Here we take the usual identification of $M \otimes \mathbb{R}$ with $\mathbb{R}^{n}$. 


\section{Untwistedness of twisted cubes and base-point free divisors}

In this section we prove the main result (Theorem 2.4) of this note, which states that the Grossberg-Karshon twisted cube is "untwisted" (i.e., the support $C(\mathbf{c}, \ell)$ is a closed, convex polytope and the support function is constant and equal to 1 on $C(\mathbf{c}, \ell)$, cf. Definition 2.2) if and only if the divisor $D(\mathbf{c}, \ell)$ of Section 1.2 is basepoint-free. Thus we are able to completely characterize the untwistedness in terms of the geometry of an associated toric variety. As a consequence, we also obtain a computationally convenient method for determining whether or not the twisted cube is untwisted (cf. Remark 2.5).

Fix a positive integer $n$. Let $\mathbf{c}=\left\{c_{i j}\right\}_{1 \leq i<j \leq n}$ and $\ell=\left\{\ell_{1}, \ldots, \ell_{n}\right\}$ be fixed integers. The following proposition gives several equivalent conditions for the untwistedness (cf. Definition 2.2) of the Grossberg-Karshon twisted cube $(C=C(\mathbf{c}, \ell), \rho)$ and additionally gives an explicit computation of $C$ in terms of toric geometry, namely, it is the polytope $P_{D(\mathbf{c}, \ell)}$. We note also that the proposition shows that if $C$ is closed, then it is automatically convex. The conditions (b) and (c) in the proposition, involving the Cartier data $\left\{m_{\sigma}\right\}$, are the most useful for later applications.

Proposition 2.1. Let $\mathbf{c}, \ell$ be as above. Let $(C(\mathbf{c}, \ell), \rho)$ denote the corresponding Grossberg-Karshon twisted polytope. Let $m_{\sigma}$ be the Cartier data of the divisor $D(\mathbf{c}, \ell)$ on $X(\mathbf{c})$ and let $P_{D(\mathbf{c}, \ell)}$ denote the associated polytope given in (1.10). Then the following are equivalent:

(a) $C=C(\mathbf{c}, \ell)$ is closed (as a subset of $\mathbb{R}^{n}$ with the usual Euclidean topology).

(b) $m_{\sigma} \in C$ for all $\sigma$.

(c) $m_{\sigma, k} \geq 0$ for all $\sigma$ and all $k$.

(d) c and $\ell$ satisfy the condition $(\mathrm{P})$.

(e) $C=P_{D(\mathbf{c}, \ell)}$.

Definition 2.2. If any of the above (equivalent) conditions hold, we say that the Grossberg-Karshon twisted cube $(C=C(\mathbf{c}, \ell), \rho)$ is untwisted. Equivalently, from condition (e) above it follows that $(C, \rho)$ is untwisted exactly when $C$ is a closed convex polytope (namely $P_{D(\mathbf{c}, \ell)}$ ) and the support for $\rho$ is constant and equal to 1 on $C$ (since $P_{D(\mathbf{c}, \ell)}$ lies in the positive orthant by definition).

In order to prove Proposition 2.1 we need a preliminary lemma.

Lemma 2.3. For all $\sigma \in\{+,-\}^{n}$, the integer vector $m_{\sigma}$ lies in the closure $\bar{C}$ of $C$ (with respect to the usual Euclidean topology).

Proof. We use induction on $n$. For the base case $n=1$, we have

$$
C=\left\{x \in \mathbb{R} \mid A_{1}=\ell_{1}<x_{1}<0 \text { or } 0 \leq x_{1} \leq A_{1}=\ell_{1}\right\} .
$$

Since $\ell_{1}$ is a fixed constant, we have that either $\ell_{1} \geq 0$ or $\ell_{1}<0$. In the case $\ell_{1} \geq 0$ we have $C=\left\{0 \leq x_{1} \leq \ell_{1}\right\}$. For $\sigma=+$ we have by (1.7) that $m_{\sigma}=0$ and for $\sigma=-$ we have $m_{\sigma}=A_{1}=\ell_{1}$. In both cases $m_{\sigma} \in C$ as desired. On 
the other hand, if $\ell_{1}<0$, then $C=\left\{\ell_{1}<x_{1}<0\right\}$ and $\bar{C}=\left\{\ell_{1} \leq x_{1} \leq 0\right\}$. A similar argument shows $m_{\sigma} \in \bar{C}$ for both $\sigma=+$ and $\sigma=-$.

Now suppose the lemma holds for $n-1$. More specifically, we assume that for any choice of vector $\sigma^{\prime}=\left(\sigma_{2}, \ldots, \sigma_{n}\right) \in\{+,-\}^{n-1}$, the vector $m_{\sigma^{\prime}}$ lies in the closure of $C(n-1)$ where $C(n-1)$ is the polytope defined in Definition 1.6. Now suppose $\sigma=\left(\sigma_{1}, \sigma_{2}, \ldots, \sigma_{n}\right) \in\{+,-\}^{n}$ and let $m_{\sigma}$ be the associated Cartier data defined by (1.7). We wish to show that $m_{\sigma}$ lies in the closure of $C$, i.e., for arbitrary fixed $\varepsilon>0$ we wish to find $x=\left(x_{1}, \ldots, x_{n}\right) \in C$ with $\left|m_{\sigma}-x\right|<\varepsilon$, where $|\cdot|$ denotes the usual Euclidean norm in $\mathbb{R}^{n}$. Let $\pi_{n-1}: \mathbb{R}^{n} \rightarrow \mathbb{R}^{n-1}$ denote the projection $\left(x_{1}, \ldots, x_{n}\right) \mapsto\left(x_{2}, \ldots, x_{n}\right)$ as in Lemma 1.7. Note that $\pi_{n-1}\left(m_{\sigma}\right)=m_{\sigma^{\prime}}$.

We first consider the case $\sigma_{1}=-$, so $m_{\sigma, 1}=A_{1}\left(m_{\sigma, 2}, \ldots, m_{\sigma, n}\right)=A_{1}\left(m_{\sigma^{\prime}}\right)$. Since the function $A_{1}\left(x_{2}, \ldots, x_{n}\right)$ is continuous, for arbitrary $\varepsilon>0$ we know there exists $\delta>0$ such that for any $x^{\prime}=\left(x_{2}, \ldots, x_{n}\right)$ with $\left|m_{\sigma^{\prime}}-x^{\prime}\right|<\delta$ then $\left|A_{1}\left(m_{\sigma^{\prime}}\right)-A_{1}\left(x^{\prime}\right)\right|=\left|m_{\sigma, 1}-A_{1}\left(x^{\prime}\right)\right|<\frac{\varepsilon}{3}$. Let $\varepsilon^{\prime}=\min \left\{\frac{\varepsilon}{3}, \delta\right\}$. By the inductive assumption we know there exists a point $x^{\prime}=\left(x_{2}, \ldots, x_{n}\right) \in C(n-1)$ such that $\left|m_{\sigma^{\prime}}-x^{\prime}\right|<\varepsilon^{\prime}$. Moreover by definition of $C$ we know $x=\left(x_{1}, \ldots, x_{n}\right) \in C$ (so $\left.\pi_{n-1}(x)=x^{\prime}\right)$ exactly if $A_{1}\left(x_{2}, \ldots, x_{n}\right)<x_{1}<0$ or $0 \leq x_{1} \leq A_{1}\left(x_{2}, \ldots, x_{n}\right)$. In particular there exists $x \in C$ with $\left|x_{1}-A_{1}\left(x_{2}, \ldots, x_{n}\right)\right|<\frac{\varepsilon}{3}$. By the triangle inequality we have

$$
\begin{aligned}
\left|x-m_{\sigma}\right| & \leq\left|x_{1}-m_{\sigma, 1}\right|+\left|x^{\prime}-m_{\sigma^{\prime}}\right| \\
& \leq\left|x_{1}-A_{1}\left(x_{2}, \ldots, x_{n}\right)\right|+\left|A_{1}\left(x_{2}, \ldots, x_{n}\right)-m_{\sigma, 1}\right|+\left|x^{\prime}-m_{\sigma^{\prime}}\right| \\
& \leq \frac{\varepsilon}{3}+\frac{\varepsilon}{3}+\frac{\varepsilon}{3}=\varepsilon,
\end{aligned}
$$

as desired.

Next we consider the case when $\sigma_{1}=+$, so $m_{\sigma, 1}=0$. From the inductive assumption we know that there exists $x^{\prime}=\left(x_{2}, \ldots, x_{n}\right) \in C(n-1)$ with $\mid m_{\sigma^{\prime}}-$ $x^{\prime} \mid<\frac{\varepsilon}{2}$. Again by definition of $C$ we know $x=\left(x_{1}, \ldots, x_{n}\right)$ is in $C$ exactly if $A_{1}\left(x_{2}, \ldots, x_{n}\right)<x_{1}<0$ or $0 \leq x_{1} \leq A_{1}\left(x_{2}, \ldots, x_{n}\right)$. In particular there exists $x \in C$ with $\left|x_{1}\right|<\frac{\varepsilon}{2}$. By the triangle inequality we have

$$
\begin{aligned}
\left|x-m_{\sigma}\right| & \leq\left|x_{1}-m_{\sigma, 1}\right|+\left|x^{\prime}-m_{\sigma^{\prime}}\right| \\
& =\left|x_{1}\right|+\left|x^{\prime}-m_{\sigma^{\prime}}\right| \\
& \leq \frac{\varepsilon}{2}+\frac{\varepsilon}{2}=\varepsilon
\end{aligned}
$$

as desired. This holds for arbitrary $\varepsilon>0$ so we conclude in both cases that $m_{\sigma} \in \bar{C}$ as was to be shown.

Proof of Proposition 2.1. The proof is by induction on the size of $n$. More specifically, we will prove first that if $n=1$, then all the statements (a) through (e) are equivalent. Then, assuming the equivalences for $n-1$, we prove the equivalences for $n$. 
First suppose $n=1$. In this case $\mathbf{c}=\left\{c_{i j}\right\}=\emptyset$ and $\ell=\left\{\ell_{1}\right\}$. From the definition of $C=C(\mathbf{c}, \ell)$ we have

$$
C=\left\{x \in \mathbb{R}: \ell_{1}<x<0 \text { or } 0 \leq x \leq \ell_{1}\right\} .
$$

In particular, it is immediate that $C$ is closed if and only if $\ell_{1} \geq 0$.

(a) $\Leftrightarrow(\mathrm{b})$ : Since $\ell_{1} \geq 0$, from the formula for $m_{\sigma}$ it follows that $m_{-}=\ell_{1}$ and $m_{+}=0$ are both in $C$. Moreover, $m_{-}$is in $C$ if and only if $\ell_{1} \geq 0$.

(a) $\Leftrightarrow(\mathrm{c})$ : Since $\ell_{1} \geq 0$ in this case, again from the definition of the $m_{\sigma}$, we see that $m_{\sigma} \geq 0$ for all $\sigma$. Moreover if $m_{-} \geq 0$, then $\ell_{1} \geq 0$.

(a) $\Leftrightarrow(\mathrm{d})$ : Condition $(\mathrm{P})$ in this case is precisely that $\ell_{1} \geq 0$.

(a) $\Leftrightarrow(\mathrm{e}):$ In this case $P_{D(\mathbf{c}, \ell)}=\left\{x \in \mathbb{R}: 0 \leq x \leq \ell_{1}\right\}$, which is equal to $C$ exactly when $\ell_{1} \geq 0$.

Assume by induction that the statements (a) through (e) are equivalent for $n-1$. We now do the rounds for $n$.

(a) $\Rightarrow$ (b). By Lemma 2.3, $m_{\sigma} \in \bar{C}$ for all $\sigma$. Since $C$ is closed, $\bar{C}=C$ and $m_{\sigma} \in C$ for all $\sigma$ as desired.

(b) $\Rightarrow$ (c). Suppose for a contradiction that there exists $\sigma$ and $k$ with $m_{\sigma, k}<$ 0. By definition of $m_{\sigma}$ this means $\sigma_{k}=-$ and $m_{\sigma, k}=A_{k}\left(m_{\sigma, k+1}, \ldots, m_{\sigma, n}\right)<$ 0 . But then $m_{\sigma, k}$ does not satisfy condition (S-k), since

$$
A_{k}\left(m_{\sigma, k+1}, \ldots, m_{\sigma, n}\right) \nless m_{\sigma, k} .
$$

Thus by definition of $C$ this implies $m_{\sigma} \notin C$, contradicting (b).

$(\mathrm{c}) \Rightarrow(\mathrm{d})$. By induction we already know that conditions (P-2) through (P$\mathrm{n})$ hold, so it suffices to check the condition (P-1). Since we assume $m_{\sigma, k} \geq 0$ for all $\sigma$ and $k$, in particular this implies that $m_{\sigma^{\prime}, k} \geq 0$ for all $\sigma^{\prime} \in\{+,-\}^{n-1}$ and $2 \leq k \leq n$. By our inductive assumption we then know that $C(2)=P_{D^{\prime}}$ where $\bar{D}^{\prime}$ is the divisor determined by $\mathbf{c}^{\prime}=\left\{c_{i j}\right\}_{2 \leq i<j \leq n}$ and $\ell^{\prime}=\left\{\ell_{2}, \ldots, \ell_{n}\right\}$. Moreover $C(2)$ is the convex hull of the $m_{\sigma^{\prime}}$ as $\sigma^{\prime}$ ranges over $\{+,-\}^{n-1}$. In particular, in this case the condition $(\mathrm{P}-1)$ is equivalent to saying that if $\left(x_{2}, \ldots, x_{n}\right) \in P_{D^{\prime}}$, then $A_{1}\left(x_{2}, \ldots, x_{n}\right) \geq 0$. By the above, this is equivalent to the statement that if $\left(x_{2}, \ldots, x_{n}\right)$ is in the convex hull of the $\left\{m_{\sigma^{\prime}}\right\}$, then $A_{1}\left(x_{2}, \ldots, x_{n}\right) \geq 0$. Since the function $A_{1}$ is linear, to check this condition it suffices to check at the vertices $\left\{m_{\sigma^{\prime}}\right\}$. For any $\sigma^{\prime} \in\{+,-\}^{n-1}$ consider $\sigma=\left(-, \sigma_{2}^{\prime}, \ldots, \sigma_{n}^{\prime}\right)$. Then $m_{\sigma, 1}=A_{1}\left(m_{\sigma^{\prime}}\right)$, but by assumption this is $\geq 0$. Hence (P-1) is satisfied, as desired.

$(\mathrm{d}) \Rightarrow(\mathrm{e})$. This is immediate from the definition of $C$ and $P_{D(\mathbf{c}, \ell)}$.

$(\mathrm{e}) \Rightarrow(\mathrm{a})$. Since $P_{D(\mathbf{c}, \ell)}$ is a closed convex polytope by definition, if $C=$ $P_{D(\mathbf{c}, \ell)}$, then in particular $C$ is closed.

The above shows that the conditions (a) through (e) are equivalent for any $n$.

The proposition above gives us many equivalent ways to check the untwistedness of the Grossberg-Karshon twisted cube, and includes some characterizations involving the Cartier data of $D(\mathbf{c}, \ell)$ on $X(\mathbf{c})$. These allow us to prove 
our main result, which connects the basepoint-freeness of the divisor $D(\mathbf{c}, \ell)$ with the untwistedness of the twisted cube.

Theorem 2.4. In the setting of Proposition 2.1, the Grossberg-Karshon twisted cube $(C(\mathbf{c}, \ell), \rho)$ is untwisted if and only if the divisor $D(\mathbf{c}, \ell)$ on $X(\mathbf{c})$ is basepoint-free. Equivalently, the twisted cube is untwisted if and only if $m_{\sigma} \in$ $P_{D(\mathbf{c}, \ell)}$ for all $\sigma$ if and only if $P_{D(\mathbf{c}, \ell)}$ is the convex hull of the $\left\{m_{\sigma}\right\}_{\sigma \in\{+,-\}^{n}}$.

Proof. It is well-known that $D(\mathbf{c}, \ell)$ is basepoint-free if and only if $m_{\sigma} \in P_{D(\mathbf{c}, \ell)}$ for all $\sigma$ if and only if $P_{D(\mathbf{c}, \ell)}$ is the convex hull of the $\left\{m_{\sigma}\right\}_{\sigma \in\{+,-\}^{n}}[3$, Theorem 6.1.7]. If $m_{\sigma} \in P_{D(\mathbf{c}, \ell)}$ for all $\sigma$, then since $P_{D(\mathbf{c}, \ell)}$ is by definition a subset of $C$ from Proposition 2.1 we may conclude that $(C, \rho)$ is untwisted. On the other hand, if $C$ is untwisted, then the conditions (b) and (e) of Proposition 2.1 holds which together these imply that $m_{\sigma} \in P_{D(\mathbf{c}, \ell)}$ for all $\sigma$ and thus $D(\mathbf{c}, \ell)$ is base-point free.

Remark 2.5. In practice, the condition which is useful for computations is Proposition 2.1(c). Indeed, the condition Proposition 2.1(c) is used in [5] in which the special case of twisted cubes arising from representation theory is studied in more detail.

Example 2.6. Let $\mathbf{c}=\left\{c_{12}=2\right\}$ and $\ell=\left\{\ell_{1}=4, \ell_{2}=3\right\}$. The linear functions $A_{1}$ and $A_{2}$ are

$$
A_{2}=3, \quad A_{1}\left(x_{2}\right)=4-2 x_{2} .
$$

Since $A_{1}(3)=-2<0$, c and $\ell$ do not satisfy the condition $(P)$. In this example we also have $D(\mathbf{c}, \ell)=4 D_{e_{1}^{-}}+3 D_{e_{2}^{-}}$and $P_{D(\mathbf{c}, \ell)}=\left\{\left(x_{1}, x_{2}\right) \in \mathbb{R}^{2} \mid 0 \leq x_{2} \leq\right.$ $\left.3,0 \leq x_{1} \leq 4-2 x_{2}\right\}$. For the maximal cone $\sigma=(-,-)$ spanned by the ray $e_{1}^{-}$, and $e_{2}^{-}$, we see that $m_{\sigma}=(-2,3) \notin P_{D}$. Thus $D(\ell, \mathbf{c})$ is not base-point free.

\section{Untwistedness vs. convexity via positivity of the $\ell_{k}$}

As Example 1.4 shows, the untwistedness of the twisted cube is not equivalent to the convexity of the twisted cube, i.e., a twisted cube may be convex but not untwisted. In this section we study their relation via the positivity of the collection of integers $\ell_{1}, \ldots, \ell_{n}$, which in turn relates to the effectivity of the divisor $D(\mathbf{c}, \ell)$.

We begin with the following observation.

Lemma 3.1. Let $n$ be a positive integer and $\mathbf{c}=\left\{c_{i j}\right\}, \ell=\left\{\ell_{1}, \ldots, \ell_{n}\right\}$ be fixed integers. If the Grossberg-Karshon twisted cube $(C=C(\mathbf{c}, \ell), \rho)$ is untwisted, then all $\ell_{i} \geq 0$.

Proof. If the Grossberg-Karshon twisted cube is untwisted, then by Theorem 2.4 we know condition $(\mathrm{P})$ holds. So we wish to show that if condition $(\mathrm{P})$ holds for $\mathbf{c}$ and $\ell$, then $\ell_{i} \geq 0$ for all $i$.

We prove this by induction on the size of $n$. Suppose $n=1$. In this case the condition $(\mathrm{P})$ consists of the single statement $(\mathrm{P}-1)$ that $\ell_{1} \geq 0$. Now suppose 
the statement holds for $n-1$. In particular, since condition $(\mathrm{P})$ includes the $n-1$ statements (P-2) to (P-n), we know that $\ell_{i} \geq 0$ for all $i$ with $2 \leq i \leq n$. In particular this means that the point $\left(x_{2}, \ldots, x_{n}\right)=(0,0, \ldots, 0)$ satisfies the hypothesis of condition (P-1), since in this case $A_{k}\left(x_{k+1}, \ldots, x_{n}\right)=\ell_{k} \geq 0$ and $x_{k}=0 \leq \ell_{k}$ for all $k$ with $2 \leq k \leq n$. Then the conclusion of (P-1) states that $A_{1}\left(x_{2}, \ldots, x_{n}\right)=A_{1}(0,0, \ldots, 0)=\ell_{1}$ must be non-negative, as desired.

The theorem below shows that in our setting, if the divisor $D(\mathbf{c}, \ell)$ is basepoint-free, then it is also effective. We also prove a partial converse.

Theorem 3.2. Let $n$ be a positive integer and let $\mathbf{c}=\left\{c_{i j}\right\}$ and $\ell=\left(\ell_{k}\right)$ be fixed integers.

(a) If the Grossberg-Karshon twisted cube $(C=C(\mathbf{c}, \ell), \rho)$ is untwisted, then $C(\mathbf{c}, \ell)$ is convex and $\ell_{k} \geq 0$ for all $k$. Equivalently, if $D(\mathbf{c}, \ell)$ is basepoint-free, then it is also effective.

(b) If $C(\mathbf{c}, \ell)$ is convex and $\ell_{k}$ is strictly positive for all $k$, then the twisted cube $(C(\mathbf{c}, \ell), \rho)$ is untwisted (equivalently, $D(\mathbf{c}, \ell)$ is base-point free).

Proof. If the twisted cube is untwisted, then by Theorem 2.4 we know that $C=C(\mathbf{c}, \ell)=P_{D(\mathbf{c}, \ell)}$ and thus $C$ is convex. Then by Lemma 3.1 above, we see all $\ell_{i}$ are non-negative.

Next suppose that $C$ is convex and all $\ell_{k}$ are strictly positive. Suppose in order to obtain a contradiction that $(C=C(\mathbf{c}, \ell), \rho)$ is not untwisted. By Theorem 2.4 this means that $C \neq P_{D}$. Since by definition $P_{D}$ is always a subset of $C$, this means that there exists a point $a=\left(a_{1}, \ldots, a_{n}\right)$ in $C \backslash P_{D}$. Since $a \notin P_{D}$, we know there exists some $j$ such that $a_{j}<0$. On the other hand, $a \in C$ so we must have $A_{j}\left(a_{j+1}, \ldots, a_{n}\right)<a_{j}<0$.

Now let $\Gamma=\left\{A_{j}(x)<x_{j}<0\right\}$ be the intersection of the two open half spaces $\left\{A_{j}(x)<x_{j}\right\}$ and $\left\{x_{j}<0\right\}$. Also let $\Gamma^{\prime}=\left\{0 \leq x_{j} \leq A_{j}(x)\right\}$ be the intersection of the two closed half spaces $\left\{0 \leq x_{j}\right\}$ and $\left\{x_{j} \leq A_{j}(x)\right\}$. Then by definition $C \subset \Gamma \cup \Gamma^{\prime}$. Note that $a \in \Gamma$. Also note that since all $\ell_{i}$ are positive, the origin $(0,0, \ldots, 0)$ is contained in $C$. Since $C$ is in addition assumed to be convex, we know that $t a \in C$ for all $t \in[0,1]$. In fact, since $a \in \Gamma$, we further know that $t a \in C \cap \Gamma$ for any $t$ with $0<t \leq 1$. Thus

$$
A_{j}(t a)-t a_{j}<0 \Leftrightarrow \ell_{j}-\sum_{k>j} c_{j k} t a_{k}-t a_{j}<0 \Leftrightarrow \ell_{j}<t\left(a_{j}+\sum_{k>j} c_{j k} a_{k}\right) .
$$

However, since by assumption $\ell_{j}>0$, the last condition in the string of equivalences above implies that the expression $a_{j}+\sum_{k>j} c_{j k} a_{k}$ must be strictly positive. Thus

$$
t a \in C \cap \Gamma^{-} \Rightarrow t>\frac{\ell_{j}}{a_{j}+\sum_{k>j} c_{j k} a_{k}} .
$$

This is a contradiction, since we may choose $t$ to be arbitrarily close to 0 . 


\section{Connection to flag varieties $G / B$}

The study of twisted polytopes initiated by Grossberg and Karshon's work [4] was motivated by representation theory and, in particular, the search for polytopes whose lattice points encode the characters of representations. In this section we record some initial observations concerning the untwistedness of the Grossberg-Karshon twisted cube when the constants $\mathbf{c}=\left\{c_{i j}\right\}$ and $\ell=\left\{\ell_{k}\right\}$ are determined from certain representation-theoretic data.

Following [4], we let $G$ be a complex semisimple simply-connected linear algebraic group of rank $r$. Choose a Cartan subgroup $H \subset G$, and a Borel subgroup $B \supset H$. Let $\alpha_{i}$ denote the simple roots, $\alpha_{i}^{\vee}$ the coroots, and $\varpi_{i}$ the fundamental weights (characterized by the relation $\left\langle\varpi_{i}, \alpha_{j}^{\vee}\right\rangle=\delta_{i j}$ ). Recall that the simple reflections $s_{\beta}: X \rightarrow X, \lambda \mapsto \lambda-\left\langle\lambda, \beta^{\vee}\right\rangle \beta$ generate the Weyl group $W$, where we let $\beta$ range among the simple roots $\alpha_{1}, \ldots, \alpha_{r}$.

Fix a choice $\lambda=\lambda_{1} \varpi_{1}+\ldots+\lambda_{r} \varpi_{r}$ in the weight lattice, where $\lambda_{i} \in \mathbb{Z}$. Also fix a choice $\underline{w}=s_{\beta_{1}} \cdots s_{\beta_{n}}$ of decomposition of an element $w$ of $W$. For such $\lambda$ and $\underline{w}$ we define constants $\mathbf{c}, \ell$ by the formulas (cf. $[4, \S 3.7]$ )

$$
c_{i j}=\left\langle\beta_{j}, \beta_{i}^{\vee}\right\rangle
$$

for $1 \leq i<j \leq n$, and

$$
\ell_{1}=\left\langle\lambda, \beta_{1}^{\vee}\right\rangle, \ldots, \ell_{n}=\left\langle\lambda, \beta_{n}^{\vee}\right\rangle .
$$

Note that if the $j$-th simple reflection in the given word decomposition $\underline{w}$ is equal to $\alpha_{i}$, then $\ell_{j}=\lambda_{i}$.

The following simple example illustrates these definitions.

Example 4.1. Consider $G=S L(3, \mathbb{C})$ with positive roots $\left\{\alpha_{1}, \alpha_{2}\right\}$. Let $\lambda=$ $2 \varpi_{1}+\varpi_{2}$ and $\underline{w}=s_{\alpha_{1}} s_{\alpha_{2}} s_{\alpha_{1}}$. Then $\left(\beta_{1}, \beta_{2}, \beta_{3}\right)=\left(\alpha_{1}, \alpha_{2}, \alpha_{1}\right)$ and we have

$$
\begin{aligned}
c_{12} & =\left\langle\alpha_{2}, \alpha_{1}^{\vee}\right\rangle=-1, \\
c_{13} & =\left\langle\alpha_{1}, \alpha_{1}^{\vee}\right\rangle=2, \\
c_{23} & =\left\langle\alpha_{1}, \alpha_{2}^{\vee}\right\rangle=-1, \\
\ell=\left(\ell_{1}, \ell_{2}, \ell_{3}\right) & =\left(\left\langle\lambda, \alpha_{1}^{\vee}\right\rangle=2,\left\langle\lambda, \alpha_{2}^{\vee}\right\rangle=1,\left\langle\lambda, \alpha_{1}^{\vee}\right\rangle=2\right) .
\end{aligned}
$$

As mentioned in the introduction, in the setting above Grossberg and Karshon derive a Demazure-type character formula for the irreducible $G$-representation corresponding to $\lambda$, expressed as a sum over the lattice points $\mathbb{Z}^{n} \cap C(\mathbf{c}, \ell)$ in the Grossberg-Karshon twisted cube $(C(\mathbf{c}, \ell), \rho)[4$, Theorem 5 and Theorem 6]. The lattice points appear with a plus or minus sign according the density function $\rho$. Hence their formula is a positive formula if $\rho$ is constant and equal to 1 on all of $C(\mathbf{c}, \ell)$. From the point of view of representation theory it is therefore of interest to determine conditions on the weight $\lambda$ and the word decomposition $\underline{w}=s_{\beta_{1}} \cdots s_{\beta_{n}}$ such that the associated Grossberg-Karshon twisted cube is in fact untwisted. The main result of this section, Theorem 4.2 below, gives 2 simple criteria which are necessary 
for untwistedness. A more detailed analysis of this situation, which gives both necessary and sufficient conditions, is in [5].

Theorem 4.2. Let $\lambda$ and $\underline{w}$ be as above and let $\mathbf{c}$ and $\ell$ be defined from $\lambda$ and $\underline{w}$ as in (4.1) and (4.2). If the corresponding Grossberg-Karshon twisted cube $(C(\mathbf{c}, \ell), \rho)$ is untwisted, then:

(1) If $\alpha_{i}$ appears in the word decomposition $\underline{w}$, then $\lambda_{i} \geq 0$. In particular, if all the simple roots $\left\{\alpha_{1}, \ldots, \alpha_{r}\right\}$ appear at least once in $\underline{w}$, then $\lambda$ is dominant.

(2) If $\alpha_{i}$ appears more than once in $\underline{w}$, then $\lambda_{i}=0$.

Proof. Since we assume that $(C(\mathbf{c}, \ell), \rho)$ is untwisted, from Theorem 3.2 we know that $\ell_{j} \geq 0$ for all $1 \leq j \leq n$. By the definition of the $\ell_{j}$ in (4.2), the first statement immediately follows.

Now suppose $\alpha_{i}$ appears more than once in $\underline{w}$, i.e., there exist distinct $j<k$ with $\beta_{j}=\beta_{k}=\alpha_{i}$. Consider the point $\left(x_{j+1}, \ldots, x_{k}, \ldots, x_{n}\right)=$ $\left(0, \ldots, \ell_{k}, \ldots, 0\right)$ where all coordinates are 0 except the $x_{k}$ coordinate, which is equal to $\ell_{k}=\lambda_{i}$. By assumption, $(C(\mathbf{c}, \ell), \rho)$ is untwisted, so condition $(\mathrm{P})$ holds. We claim that the chosen point $\left(x_{j+1}, \ldots, x_{k}, \ldots, x_{n}\right)$ satisfies the hypotheses in the statement of condition $(\mathrm{P}-\mathrm{j})$. To see this, first observe that the last $n-k$ coordinates are all 0 and $A_{k}(0, \ldots, 0)=\ell_{k}$, so the last $n-k+1$ coordinates satisfy the hypotheses for condition $(\mathrm{P}-(\mathrm{k}-1))$. The conclusion of $(\mathrm{P}-(\mathrm{k}-$ $1))$ then states that $A_{k-1}\left(\ell_{k}, 0, \ldots, 0\right) \geq 0$. Hence $\left(x_{k-1}=0, x_{k}=\ell_{k}, 0, \ldots, 0\right)$ satisfies the hypotheses for the next condition $(\mathrm{P}-(\mathrm{k}-2))$. Proceeding similarly shows that $\left(x_{j+1}, \ldots, x_{k}, \ldots, x_{n}\right)$ satisfies the hypotheses of $(\mathrm{P}-\mathrm{j})$. Then the conclusion of $(\mathrm{P}-\mathrm{j})$ states that $A_{j}\left(0, \ldots, \ell_{k}, 0 \ldots, 0\right) \geq 0$. By definition

$$
\begin{aligned}
A_{j}\left(0, \ldots, \ell_{k}, \ldots, 0\right) & =\ell_{j}-c_{j k} x_{k} \\
& =\ell_{j}-\left\langle\beta_{k}, \beta_{j}^{\vee}\right\rangle \ell_{k} \\
& =\lambda_{i}-\left\langle\alpha_{i}, \alpha_{i}^{\vee}\right\rangle \lambda_{i} \\
& =\lambda_{i}-2 \lambda_{i} \\
& =-\lambda_{i} .
\end{aligned}
$$

Hence $\lambda_{i} \leq 0$. But we already knew $\lambda_{i} \geq 0$, so we conclude $\lambda_{i}=0$, as required.

The converse of Theorem 4.2 does not hold, as can be seen by the following. We thank Eunjeong Lee for providing this example.

Example 4.3. Consider $G=\mathrm{SL}_{4}(\mathbb{C})$. Choose $\underline{w}=s_{\alpha_{2}} s_{\alpha_{1}} s_{\alpha_{2}} s_{\alpha_{3}} s_{\alpha_{2}} s_{\alpha_{1}}$ and $\lambda=2 \varpi_{3}$. Then the corresponding choices of constants are

$$
\begin{aligned}
& \begin{array}{llllllllll}
c_{12} & c_{13} & c_{14} & c_{15} & c_{16} & -1 & 2 & -1 & 2 & -1
\end{array} \\
& \begin{array}{llllllll}
c_{23} & c_{24} & c_{25} & c_{26} & -1 & 0 & -1 & 2
\end{array}
\end{aligned}
$$

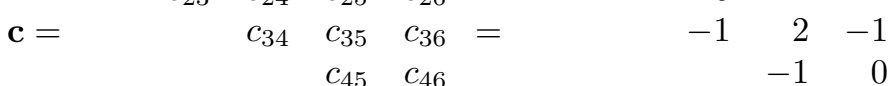

$$
\begin{aligned}
& \begin{array}{ll}
c_{56} & -1
\end{array}
\end{aligned}
$$


and

$$
\ell=\ell_{1} \quad \ell_{2} \quad \ell_{3} \quad \ell_{4} \quad \ell_{5} \quad \ell_{6}=\begin{array}{lllllll}
0 & 0 & 0 & 2 & 0 & 0
\end{array} .
$$

The linear functions $A_{j}$ arising in the definition of the associated twisted cube are given by

$$
\begin{aligned}
& A_{1}=\begin{array}{llllll}
0 & +x_{2} & -2 x_{3} & +x_{4} & -2 x_{5} & +x_{6}
\end{array} \\
& A_{2}=\begin{array}{llll}
0 & +x_{3} & +x_{5} & -2 x_{6}
\end{array} \\
& A_{3}=\begin{array}{llll}
0 & +x_{4} & -2 x_{5} & +x_{6}
\end{array} \\
& \begin{array}{llll}
A_{4} & =2 & +x_{5} & \\
A_{5} & =0 & & x_{6}
\end{array} \\
& A_{6}=0
\end{aligned}
$$

It can now be seen that for the maximal cone $\sigma=(-,-,-,-,-,-)$, the corresponding Cartier data $m_{\sigma}=(-2,2,2,2,0,0)$ is not contained in $P_{D(\mathbf{c}, \ell)}$. Thus by Theorem 2.4, the Grossberg-Karshon twisted cube is not untwisted in this case, despite the fact that both of the conditions stated in Theorem 4.2 are satisfied.

\section{References}

[1] V. Alexeev and M. Brion, Toric degenerations and spherical varieties, Selecta Math. 10 (2004), no. 4, 453-478.

[2] D. Anderson, Okounkov bodies and toric degenerations, Math. Ann. 356 (2013), no. 3, 1183-1202.

[3] D. Cox and J. Little, and H. Schenck, Toric Varieties, Graduate Studies in Mathematics, 124. American Mathematical Society, Providence, RI, 2011.

[4] M. Grossberg and Y. Karshon, Bott towers, complete integrability, and the extended character of representations, Duke Math. J. 76 (1994), no. 1, 23-58.

[5] M. Harada and E. Lee, Grossberg-Karshon twisted cubes and hesitant walk avoidance, Arxiv:1407.8543.

[6] K. Kaveh, Crystal bases and Newton-Okounkov bodies, Arxiv:1101.1687.

[7] V. Kiritchenko, Divided difference operators on polytopes, Arxiv:1307.7234.

[8] B. Pasquier, Vanishing theorem for the cohomology of line bundles on Bott-Samelson varieties, J. Algebra 323 (2010), no. 10, 2834-2847.

Megumi Harada

Department of Mathematics and Statistics

MCMASTER UNIVERSITY

1280 Main Street West

Hamilton, Ontario L8S4K1, Canada

E-mail address: Megumi.Harada@math.mcmaster.ca

JiHyEON JeSSIE YANG

Department of Mathematics and Statistics

MCMASTER UNIVERSITY

1280 Main Street West

Hamilton, Ontario L8S4K1, Canada

E-mail address: jyang@math.mcmaster.ca 